\title{
POPULATION STUDY ARTICLE US adolescents at risk for not meeting physical activity recommendations by season
}

\author{
Melanie L. Kornides ${ }^{1}$, Matthew W. Gillman ${ }^{2,3}$, Bernard Rosner ${ }^{4,5}$, Eric B. Rimm ${ }^{3,4,6}$, Jorge E. Chavarro ${ }^{3,4,6}$ and Alison E. Field $^{7}$
}

\begin{abstract}
BACKGROUND: We sought to identify regional and seasonal variation in not meeting physical activity (PA) recommendations of $\geq 60 \mathrm{~min}$ a day of moderate-to-vigorous PA (MVPA) and $3 \mathrm{~h}$ of vigorous PA per week (VPA) in a longitudinal cohort of United States (US) adolescents.

METHODS: Participants in the Growing Up Today Study 2, a prospective study of 10,918 adolescents, self-reported season-specific weekly hours of MVPA and VPA from 2004 through 2011. To assess variation in PA by climate, we grouped the contiguous US into nine climatically consistent geographic regions. We also examined MVPA and VPA by season, sex, ethnicity, weight status, and age group.

RESULTS: The majority (85\%) of adolescents did not meet the MVPA recommendation, and $91 \%$ did not meet the VPA recommendation, for one or more seasons over the four study years. Across all climate regions, adolescents were two times more likely to not meet the MVPA recommendation during the winter compared to summer (odds ratio $2.02,95 \%$ confidence interval: 1.96-2.08).

CONCLUSION: Regardless of climate region, gender, ethnicity, or age group, adolescents were more likely not to meet MVPA or VPA recommendations in the winter than the summer. Adolescents may benefit from interventions aimed at increasing PA in the winter.
\end{abstract}

Pediatric Research (2018) 84:50-56; https://doi.org/10.1038/s41390-018-0024-x

\section{INTRODUCTION}

Public health experts recommend that children and adolescents engage in $\geq 60 \mathrm{~min}$ per day of physical activity (PA). ${ }^{1}$ Of the accumulated 7 or more hours each week, at least $3 \mathrm{~h}$ should include vigorous PA (VPA), and the remaining should include moderate-or-vigorous PA (MVPA). To estimate the prevalence of adolescents meeting these PA recommendations, researchers typically measure PA cross-sectionally. However, PA may vary by season of the year, region of the country, changes in climate, and seasonal sports participation. ${ }^{2-4}$ Thus, measurements from one point in time may not represent usual PA over an entire year. ${ }^{5}$ Accelerometer data collected over several days may not adequately capture important sources of variation in PA, and as a result, may overestimate or underestimate the number of adolescents who do not meet the PA recommendations depending on the season. ${ }^{2}$ An additional limitation is the inability of accelerometer measures to capture certain activities such as swimming and bicycling, resulting in a potential underestimation of PA. ${ }^{6-8}$ Repeated measures of self-reported PA can provide information on the relative differences in levels across seasons and developmental stages in large population-based cohort studies, while reducing participant burden and cost. ${ }^{9,10}$

To our knowledge, no longitudinal adolescent cohort studies have examined the seasonal variation in PA across climatically distinct geographic regions of the United States (US), as well as how factors such as sex, weight status, and developmental stage are associated with variability in meeting PA recommendations. Prior research on seasonal variation in adolescent PA have found increased levels in the spring and summer seasons as compared to the fall and winter seasons, but have been limited to specific geographic regions and age groups, thus their generalizability is unknown. ${ }^{2,4}$ Our objective was to prospectively assess the patterns and regional trends in self-reported, season-specific PA in a longitudinal cohort of US adolescents. To determine subgroups of adolescents at risk for not meeting PA recommendations, we sought to identify the factors associated with increased odds of not meeting the respective MVPA and VPA recommendations, defined as $<60 \mathrm{~min}$ per day for MVPA, and including $<3 \mathrm{~h}$ per week of VPA.

\section{METHODS}

Study population

Participants from the Growing Up Today Study 2 (GUTS 2), a longitudinal cohort of offspring of participants of the Nurses' Health II study, self-reported season-specific PA in 2004, 2006, 2008, and $2011 .{ }^{11}$ In 2004, 6002 girls and 4916 boys ages 9-15 years returned the baseline questionnaires and thus gave assent

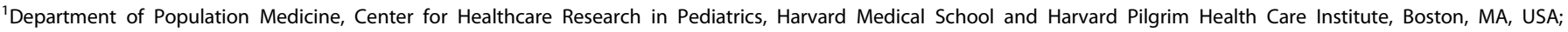

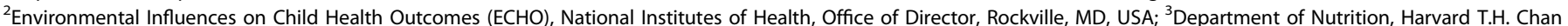

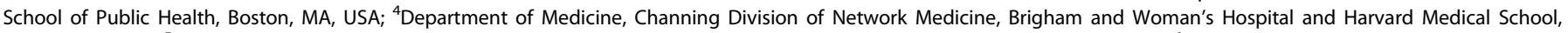

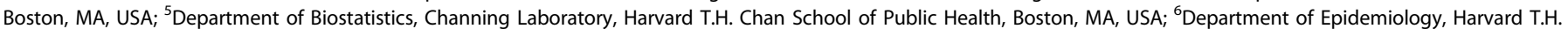
Chan School of Public Health, Boston, MA, USA and ${ }^{7}$ Department of Epidemiology, Brown University School of Public Health, Providence, RI, USA Correspondence: Melanie L. Kornides (Melanie.Kornides@mail.harvard.edu)
} 
to participate. The study was approved by the Human Subjects Committee at Brigham and Women's Hospital. Details of the study methods are described elsewhere. ${ }^{12,13}$

Adolescents with PA data on at least one survey year were eligible for inclusion in the analysis. Participants were excluded at baseline for the following reasons: missing PA $(n=3)$, missing weight status ( $n=649)$, or living in a geographical area with too few participants to conduct analyses $(n=191)$. After these exclusions, 10,095 adolescents were eligible for the study in 2004. In a sensitivity analysis, adolescents with missing data after baseline did not differ from those who remained in the study with respect to PA levels by season, region of the country, or ethnicity. Boys were more likely to have missing data than girls, and overweight/obese adolescents were more likely to have missing data than their normal weight peers $(P<.05)$. We conducted all analyses using SAS 9.4 (Cary, NC, USA).

\section{PA assessment}

Participants reported hours per week each season (spring, summer, fall, winter) they engaged in 15 activities for girls and 16 for boys. The activities included were from a previously validated, non-seasonal questionnaire. ${ }^{14,15}$ Activities included baseball/softball, basketball, biking, dancing/aerobics, American football (boys only), ice/field/street hockey or lacrosse, running/ jogging, swimming, rollerblading/skating or ice-skating, skateboarding, soccer (association football), tennis/other racquet sports, Stairmaster/elliptical or rowing machine, gymnastics or cheerleading, strength training, and volleyball. The earlier, non-seasonal version of the PA questionnaire had moderate validity and strong reliability but was difficult for children to complete as their PA patterns changed by season over the year. ${ }^{14,15}$ The seasonal format questionnaire is easier for children to complete, with improved accuracy. ${ }^{16}$ For each survey year that an adolescent reported $\mathrm{PA}$, he or she contributed information for four personseasons. There was a maximum of 16 person-seasons of PA for each participant.

We combined hours per week in all reported sports/activities to create separate total weekly MVPA and VPA estimates for each season. We used metabolic equivalent of task (MET) to classify the intensity of each activity as either moderate-to-vigorous or vigorous. MET values are defined as the ratio of the work metabolic rate to a standard resting metabolic rate (RMR) of 1.0 $\mathrm{kcal} \mathrm{kg}^{-1} \mathrm{~h}^{-1} .{ }^{17}$ METS can be used to distinguish between lightintensity ( $<3$ METs), moderate-intensity (3-6 METs), and vigorousintensity ( $>6$ METs) activities. We assigned the "general" MET level for each sport based on the 2011 Compendium of Physical Activities because study participants did not report intensity. ${ }^{17}$ For example, METs for running can vary from 4.5 to 23.0 depending on intensity, but we assigned all running a MET level of 7.0 for "general" jogging.

To identify participants who met the MPVA recommendation of $\geq 60$ min a day, we summed the time spent in activities with a general MET level $>3$. To identify participants who met the VPA recommendation of $\geq 3 \mathrm{~h}$ a week, we summed time spent each week in activities with MET levels $>6$. VPA activities included basketball, hockey/lacrosse, running, skating, soccer, tennis, and use of aerobic exercise machines. All VPA activities were also included in the MVPA estimates. In previous comparisons of accelerometer to self-report for adolescents' activity-specific MVPA, adolescents accurately estimated activity-specific time spent in MVPA within several minutes to the accelerometerestimate apart from walking. ${ }^{6}$ The majority of accelerometercaptured time spent in walking fell below MVPA threshold. To adjust for potential overestimation of MPVA, we excluded walking from the MVPA measure. We included swimming in the MVPA but not the VPA measure due to the large variation in MET values, depending on the type of stroke and intensity of effort (range 3.5 to 10). Swimming was most popular at younger ages (ages 9-10 years) during the summer months, suggesting recreational rather than competitive swimming. We assumed a MET level of 5.8, that is, swimming laps, freestyle, and front crawl, with slow, light, or moderate effort. For the same reason, we included biking in the MVPA but not the VPA category.

\section{Correlates}

We examined whether PA levels were related to season of the year, climate region of the country, sex, age, ethnic group, weight status, and self-reported hours per week of television viewing. Region of the country was updated each survey year. We categorized the adolescents' home states into nine climate regions in the continental US. The regions consisted of groupings of states with consistently similar climates as identified by the National Climatic Data Center (www.ncdc.noaa.gov/monitoringreferences/maps/us-climate-regions.php; last accessed 7/6/2017). We excluded participants who resided outside the US $(n=131)$; due to small sample sizes, we also excluded those in Alaska $(n=$ $8)$, Hawaii $(n=10)$, and the Northern Rockies and Plains region ( $n$ $=42$ ).

Ethnicity was self-reported at baseline as white, black, Hispanic, or other. Based on the American Academy of Pediatrics classification for age groups, we assessed four age groups/ developmental stages: pre-adolescence (ages 9-10 years), early adolescence (ages 11-14 years), middle adolescence, ${ }^{15-17}$ and late adolescence (ages 18-21 years). ${ }^{18}$ We calculated body mass index (BMI) $\left(\mathrm{kg} / \mathrm{m}^{2}\right)$ from self-reported height and weight. Self-reported height and weight correlate strongly with objectively measured anthropometrics in adolescents. ${ }^{19}$ We used the International Obesity Task Force BMI cut-offs for children and adolescents to classify participants as overweight/obese vs. healthy or underweight. $^{20}$

\section{Statistical analyses}

We dichotomized weekly hours of PA to indicate not meeting vs. meeting the recommendations for MVPA and VPA. To assess the odds of not meeting the respective MVPA and VPA recommendations, we fit binary logistic regression models using generalized estimating equations with an independent correlation working structure to account for the correlations between siblings and between the repeated measures over time for each individual. An increased odds ratio $(O R>1)$ represents the odds of not meeting either MVPA or VPA recommendations, respectively, as compared to meeting the recommendation.

We selected the reference groups for the correlates a priori as those groups we hypothesized to have the highest levels of PA based on prior literature. By using the group with the highest PA level as the referent, we could identify subgroups potentially at increased risk for not meeting MVPA and VPA recommendations.

To assess for group differences within individual seasons (effect modification by season), we stratified the described models by season. We had inadequate power to assess pre-adolescent PA separately. Because pre-adolescents followed similar PA patterns to those in the early adolescent stage, we compared models excluding pre-adolescents to those combining the pre-adolescent and early adolescent age groups. As there were not substantial differences, we combined the pre-adolescent and early adolescent age groups in the final models. We excluded self-reported time spent watching television each week from the final models as it was not significantly associated with PA $(P>.05)$, nor did it substantially alter the associations between PA and the other variables.

As a sensitivity analysis, we fit the same models using generalized linear mixed effects models for binary outcomes; we obtained very similar estimates (results not shown). In a secondary analysis, we also modeled continuous PA (hours per week) using longitudinal linear mixed effects models. To reduce the influence of implausible values of PA due to the right skew of both the 
MVPA and VPA hours, we set all PA for adolescents reporting $>40$ $\mathrm{h}$ a week to a maximum of $40 \mathrm{~h}$. All analyses were completed using SAS 9.4 (Cary, NC, USA). Statistical tests were two-tailed with a critical $a$ of 0.05 unless otherwise noted.

\section{RESULTS}

The sample characteristics are presented in Table 1. The majority of adolescents (85\%, $n=8579)$ did not meet the MVPA recommendation of $\geq 60 \mathrm{~min}$ per day and $91 \%(n=9231)$ did not include $\geq 3 \mathrm{~h}$ per week of VPA during one or more of the 16 study seasons. The number of adolescents who did not meet the respective recommendations varied by gender, weight status, age group, season of the year, and region of the country. The majority of girls $(87 \%, n=4834)$ and boys $(82 \%, n=3745)$ did not meet the MVPA recommendation for one or more seasons. In addition, $93 \%$ of girls $(n=5155)$ and $89 \%$ of boys $(n=4076)$ did not meet the VPA recommendation for one or more seasons. After adjusting for age, ethnicity, weight status, region of the country, girls had $40 \%$ higher odds of not meeting the MVPA recommendation (odds ratio (OR) 1.40, 95\% confidence interval Cl: 1.33-1.47) and $46 \%$ higher odds of not meeting the VPA recommendation (OR 1.46, 95\% Cl: 1.39-1.54) compared to boys (Table 2). Overweight or obese adolescents had $22 \%$ higher odds of not meeting the MVPA recommendation compared to their normal weight peers (OR 1.22, 95\% Cl: 1.16-1.28).

Among the pre-adolescent, early adolescent, and middle adolescent age groups, there was considerable variation in the percentage of the cohort not meeting the MVPA recommendation when assessed within each season (Fig. 1). Younger adolescents reported higher levels of PA in the summers: pre-adolescents and early adolescents (ages 9-14 years) had 11\% lower odds of not meeting the MVPA recommendation in the summer compared to those ages 15-17 years (Table 3, OR 0.89, 95\% Cl: 0.84-0.94). During the winter, pre-adolescents and early adolescents had 1.62 times the odds of not meeting the MVPA recommendation compared to middle adolescents (OR 1.62, 95\% Cl: 1.53-1.72).

Table 1. Baseline characteristics of the Growing Up Today Study 2 Cohort $(n=10,095)^{\mathrm{a}}$

\begin{tabular}{|c|c|c|}
\hline & $\begin{array}{l}\text { Girls }(n=5521) \\
n(\%)\end{array}$ & $\begin{array}{l}\text { Boys }(n=4574) \\
n(\%)\end{array}$ \\
\hline \multicolumn{3}{|l|}{ Age groups (years) } \\
\hline Pre-adolescent (9-10) & $544(10)$ & $505(11)$ \\
\hline Early adolescent (11-14) & $3373(61)$ & $2930(64)$ \\
\hline Middle adolescent (15-17) & $1604(29)$ & $1139(25)$ \\
\hline \multicolumn{3}{|l|}{ Climate region } \\
\hline South & $304(6)$ & $262(6)$ \\
\hline Southeast & $398(7)$ & $337(7)$ \\
\hline Southwest & $59(1)$ & $56(1)$ \\
\hline West & $718(13)$ & $605(13)$ \\
\hline Northwest & $56(1)$ & $44(1)$ \\
\hline Midwest & $855(16)$ & $704(15)$ \\
\hline Ohio Valley & $1296(24)$ & $1037(23)$ \\
\hline Northeast & $1835(33)$ & $1529(33)$ \\
\hline \multicolumn{3}{|l|}{ Ethnicity } \\
\hline White, non-Hispanic & 5114 & 4307 \\
\hline Other $^{b}$ & 407 & 266 \\
\hline Overweight/obese ${ }^{c}$ & 1039 & 1155 \\
\hline \multicolumn{3}{|c|}{$\begin{array}{l}\text { a } \text { Of the original } 10,918 \text { adolescents enrolled at baseline, } 10,095 \text { were } \\
\text { eligible for present study after exclusion for missing exposure }(n=3) \\
\text { outcome ( } n=649) \text {, or small geographic region }(n=191) \\
\text { b Other ethnicity: Black, Hispanic, Asian, Pacific Islander, Native American, } \\
\text { and unreported } \\
{ }^{c} \text { Overweight/obese defined by International Obesity Task Force BMI cut- } \\
\text { points for adolescents by age and sex }\end{array}$} \\
\hline
\end{tabular}

Table 2. ORs for not meeting MVPA and VPA recommendations ( $n=$ $10,918)$

\begin{tabular}{|c|c|c|}
\hline & $\begin{array}{l}\text { MVPA } \\
\text { OR (95\% Cl) }\end{array}$ & $\begin{array}{l}\text { VPA } \\
\text { OR }(95 \% \mathrm{Cl})\end{array}$ \\
\hline Survey year & $1.02(1.01-1.03)$ & $1.00(0.99-1.02$ \\
\hline \multicolumn{3}{|l|}{ Season } \\
\hline Summer & 1 & 1 \\
\hline Fall & $1.36(1.33-1.40)$ & $0.93(0.91-0.95$ \\
\hline Winter & $2.02(1.96-2.08)$ & $1.22(1.18-1.25$ \\
\hline Spring & $1.27(1.24-1.31)$ & $0.90(0.88-0.92$ \\
\hline \multicolumn{3}{|l|}{ Age groups (years) } \\
\hline Middle adolescent (15-17) & 1 & 1 \\
\hline Pre-adolescent (9-10)/early adolescent (11-14) & $1.22(1.17-1.27)$ & $1.05(1-1.10)$ \\
\hline Late adolescent $(\geq 18)$ & $1.50(1.42-1.58)$ & $1.08(1.02-1.14$ \\
\hline \multicolumn{3}{|l|}{ Weight status } \\
\hline Normal & 1 & 1 \\
\hline Overweight /obese & $1.22(1.16-1.28)$ & $1.32(1.25-1.39$ \\
\hline \multicolumn{3}{|l|}{ Climate region } \\
\hline Northeast & 1 & 1 \\
\hline South & $1.35(1.21-1.51)$ & $1.38(1.23-1.55$ \\
\hline Southeast & $1.30(1.18-1.44)$ & $1.25(1.13-1.39$ \\
\hline Southwest & $1.10(0.89-1.37)$ & $1.38(1.09-1.74$ \\
\hline West & $1.14(1.05-1.23)$ & $1.42(1.31-1.55$ \\
\hline Northwest & $0.96(0.77-1.20)$ & $1.29(1.02-1.63$ \\
\hline Midwest & $1.06(0.98-1.14)$ & $1.09(1.01-1.17$ \\
\hline Ohio Valley & $1.12(1.05-1.20)$ & $1.19(1.11-1.28$ \\
\hline \multicolumn{3}{|l|}{ Ethnicity } \\
\hline \multicolumn{3}{|l|}{ White } \\
\hline Other & $1.12(1.01-1.24)$ & $1.03(0.94-1.15$ \\
\hline \multicolumn{3}{|l|}{ Gender } \\
\hline Boys & 1 & 1 \\
\hline Girls & $1.40(1.33-1.47)$ & $1.47(1.39-1.54$ \\
\hline
\end{tabular}

Note: MVPA recommendation: $\geq 60$ min per day, VPA recommendation: $\geq 3 \mathrm{~h}$ per week

MVPA moderate-to-vigorous physical activity, VPA vigorous physical activity, $\mathrm{OR}$ odds ratio, $\mathrm{Cl}$ confidence interval

Older adolescents (ages 18+ years) had consistently higher odds of not meeting the MVPA recommendation each season compared to the middle adolescent age group.

Adolescents reported lower PA levels during the winter, regardless of gender, age group, weight status, ethnicity, calendar year, or climate region. Seasonally, 60\% $(n=6050)$ reported not meeting the MVPA recommendation during one or more summers over the course of the study, while $78 \%(n=7913)$ reported not meeting the MVPA recommendation during one or more winters. After adjusting for age, ethnicity, weight status, region of the country and gender, participants had 2.02 times the odds of not meeting the MVPA recommendation in the winters compared to the summers (Table 2, OR: $2.02,95 \%$ Cl: 1.96-2.07). In the analyses of hours of PA, adolescents reported 3.78 fewer hours (95\% Cl: 3.88-3.68) of MVPA each week in the winters as compared to the summers (Table 4).

Regional differences in the percentage of adolescents who did not meet the MVPA recommendation were more pronounced during the summer (Fig. 2). Compared to $27 \%$ living in the Northeast $(n=923), 39 \%$ of those living in the South $(n=223)$ and $40 \%$ in the Southeast $(n=293)$ reported not meeting the MVPA recommendation in the summer of 2004. During the winter of 2004, the percentages of adolescents not meeting the MVPA recommendation were similar amongst the various regions with over $60 \%$ not meeting the recommendation in most regions. When examined within season (Table 3), those living in the South, Southeast, and West compared to the Northeast had higher odds of not meeting the MVPA recommendation each season. There were not significant differences in odds for not meeting the MVPA 
a 100

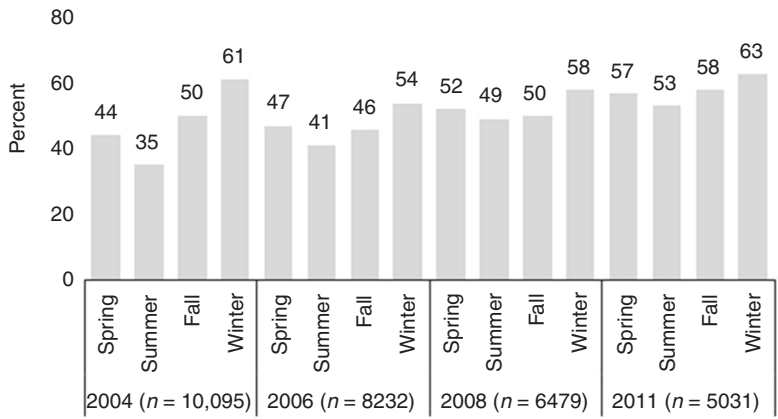

C 100

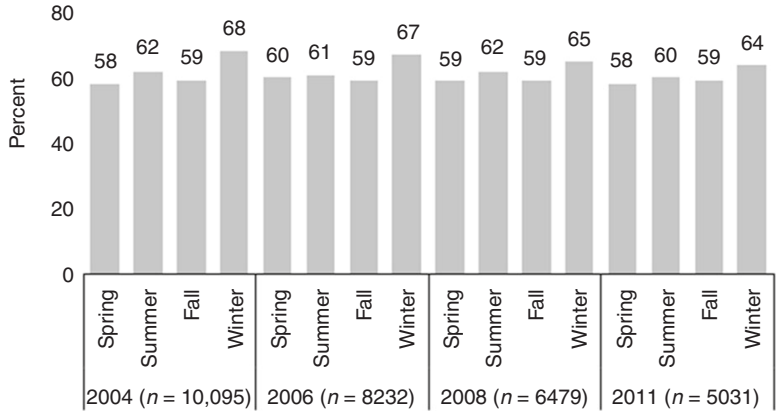

b

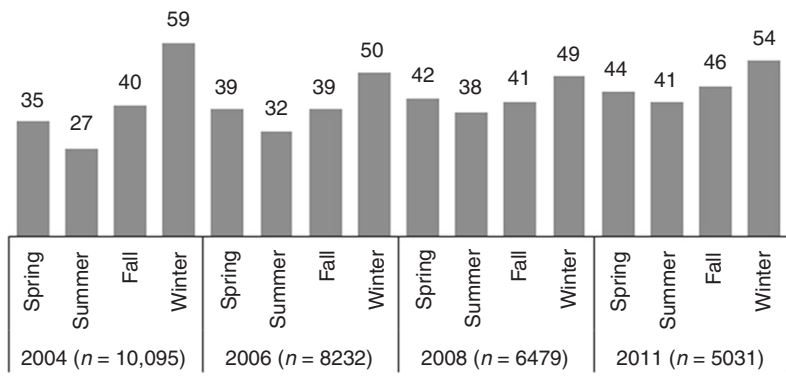

d

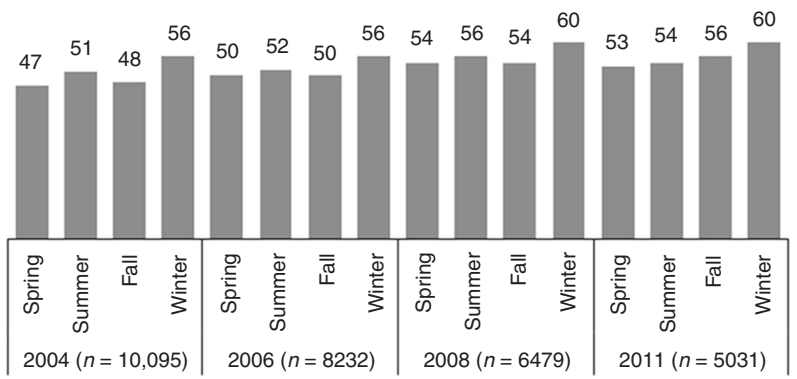

Fig. 1 Adolescents not meeting physical activity recommendations by gender, season, and year. a Percent (\%) of girls not meeting moderateto-vigorous physical activity (MVPA) recommendation of $60 \mathrm{~min}$ per day; b percent (\%) of boys not meeting MVPA recommendation; $\mathbf{c}$ percent (\%) of girls not meeting vigorous physical activity (VPA) recommendation of $3 \mathrm{~h}$ per week; d percent (\%) of boys not meeting VPA recommendation

Table 3. Season-specific ORs for not meeting MVPA recommendation $(n=10,918)$

MVPA $<60$ min per day ${ }^{\mathrm{a}}$

\begin{tabular}{|c|c|c|c|c|}
\hline & \\
\hline & $\begin{array}{l}\text { Spring } \\
\text { OR }(95 \% \mathrm{Cl})\end{array}$ & $\begin{array}{l}\text { Summer } \\
\text { OR }(95 \% \mathrm{Cl})\end{array}$ & $\begin{array}{l}\text { Fall } \\
\text { OR }(95 \% \mathrm{Cl})\end{array}$ & $\begin{array}{l}\text { Winter } \\
\text { OR }(95 \% \mathrm{Cl})\end{array}$ \\
\hline \multicolumn{5}{|l|}{ Gender } \\
\hline Girls & $1.50(1.41-1.59)$ & $1.50(1.41-1.59)$ & $1.49(1.40-1.58)$ & $1.26(1.19-1.33)$ \\
\hline \multicolumn{5}{|l|}{ Age groups (years) } \\
\hline Late adolescent $(\geq 18)$ & $1.40(1.31-1.50)$ & $1.52(1.42-1.62)$ & $1.57(1.47-1.68)$ & $1.46(1.36-1.56)$ \\
\hline \multicolumn{5}{|l|}{ Climate region } \\
\hline Northeast & 1 & 1 & 1 & 1 \\
\hline South & $1.36(1.19-1.55)$ & $1.55(1.36-1.76)$ & $1.27(1.12-1.45)$ & $1.23(1.08-1.40)$ \\
\hline Southeast & $1.26(1.12-1.42)$ & $1.43(1.27-1.61)$ & $1.25(1.11-1.41)$ & $1.21(1.08-1.37)$ \\
\hline Ohio Valley & $1.24(1.14-1.34)$ & $1.04(0.96-1.13)$ & $1.15(1.06-1.24)$ & $1.15(1.06-1.25)$ \\
\hline
\end{tabular}


Table 4. Predictors of weekly hours of MVPA and VPA (Longitudinal Linear Mixed Effects Model) $(n=10,918)$

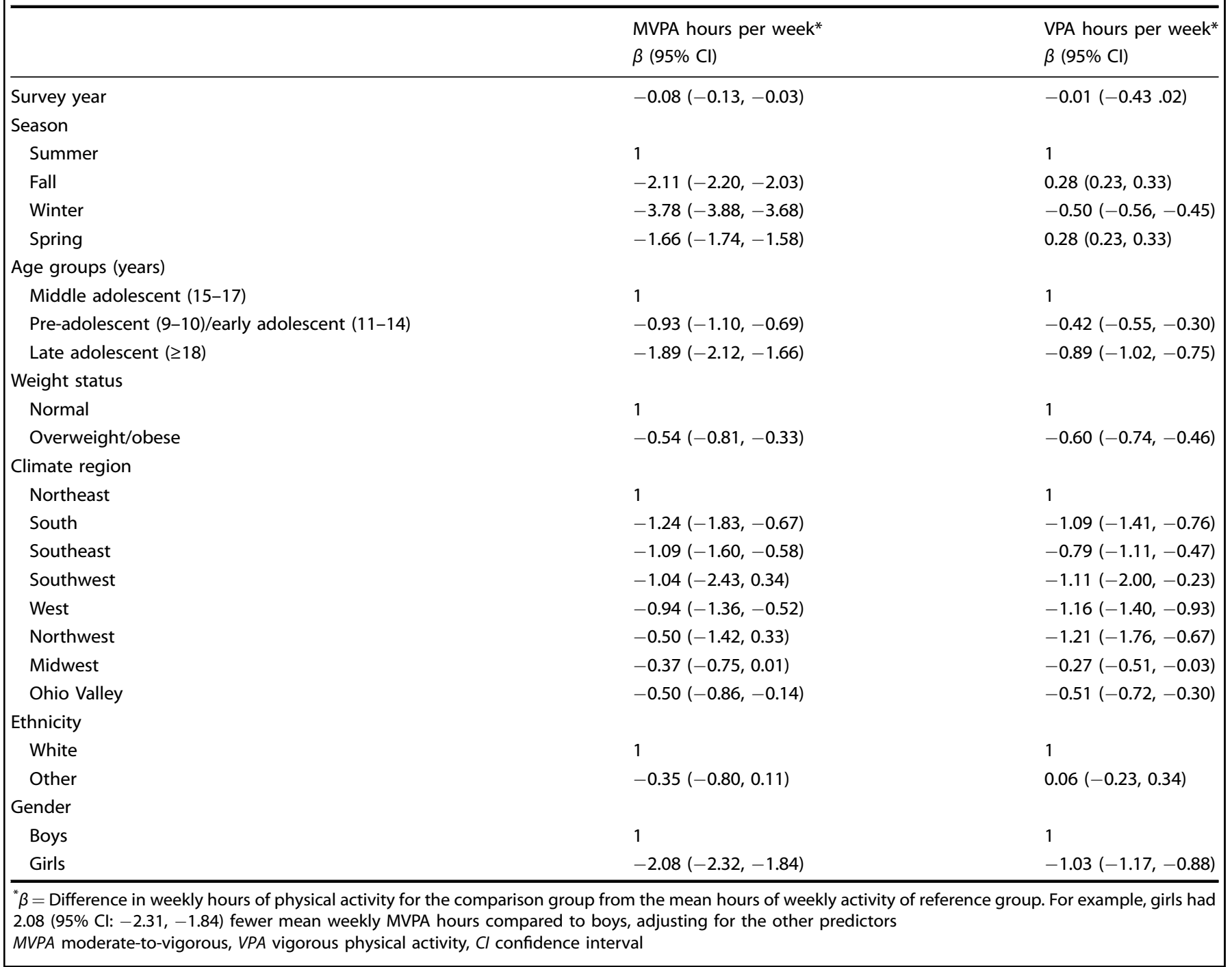

recommendation between the Northeast and the Southwest or Northwest regardless of season.

\section{DISCUSSION}

Adolescents in our prospective study reported remarkably similar patterns of higher PA in the summer and lower PA in the winter across US climate regions, even after adjusting for age, weight status, and gender. Previous findings on seasonal variation in PA amongst adolescents have been inconsistent across studies. ${ }^{21-25}$ Though it has been proposed that the lack of seasonal variability in PA found in some studies is related to their focused geographic scope in more temperate climate regions with milder winters, $2,4,26$ our findings suggests that extreme temperatures are not strong influencers on US adolescent PA patterns in general. Overall, we found that $85 \%$ of adolescents did not meet the MVPA recommendation of $\geq 60$ min per day and $91 \%$ did not meet the VPA recommendation of $\geq 3 \mathrm{~h}$ per week during one or more seasons over the four study years. These findings provide new data on how seasonal PA may vary across age, gender, weight status, and geographical region among US adolescents.

Across the US, adolescents were more active in the summers and less active in the winters, regardless of climate region.
However, we observed relatively more variation amongst climate regions in not meeting the MVPA recommendation during the summer as compared to other seasons. Adolescents who lived in the South, Southeast, and West were more likely to report not meeting the MVPA recommendation during the summers compared to those living in the Northeast. In contrast, adolescents living in the Northwest did not differ significantly from the Northeast in their PA levels or risk of not meeting PA recommendations within each season. Although we were unable to assess climate differences below the state level, our findings suggest differences in summer climates across regions do contribute to variation in the PA levels among US adolescents during that season.

Few studies of seasonal variation in adolescent PA have compared age subgroups. Overall, adolescents' PA levels generally decline with age. ${ }^{27,28}$ Consistent with this trend, PA levels declined as adolescents aged over our study period. As a notable exception, we found the middle adolescent age group (ages 15-17 years) to be the more active compared to the pre-adolescent, early adolescent and late adolescent age groups after adjusting for season and other factors. We also observed larger seasonal variations in PA within the younger age groups, and less seasonal variation in PA levels for older adolescents and young adults, 


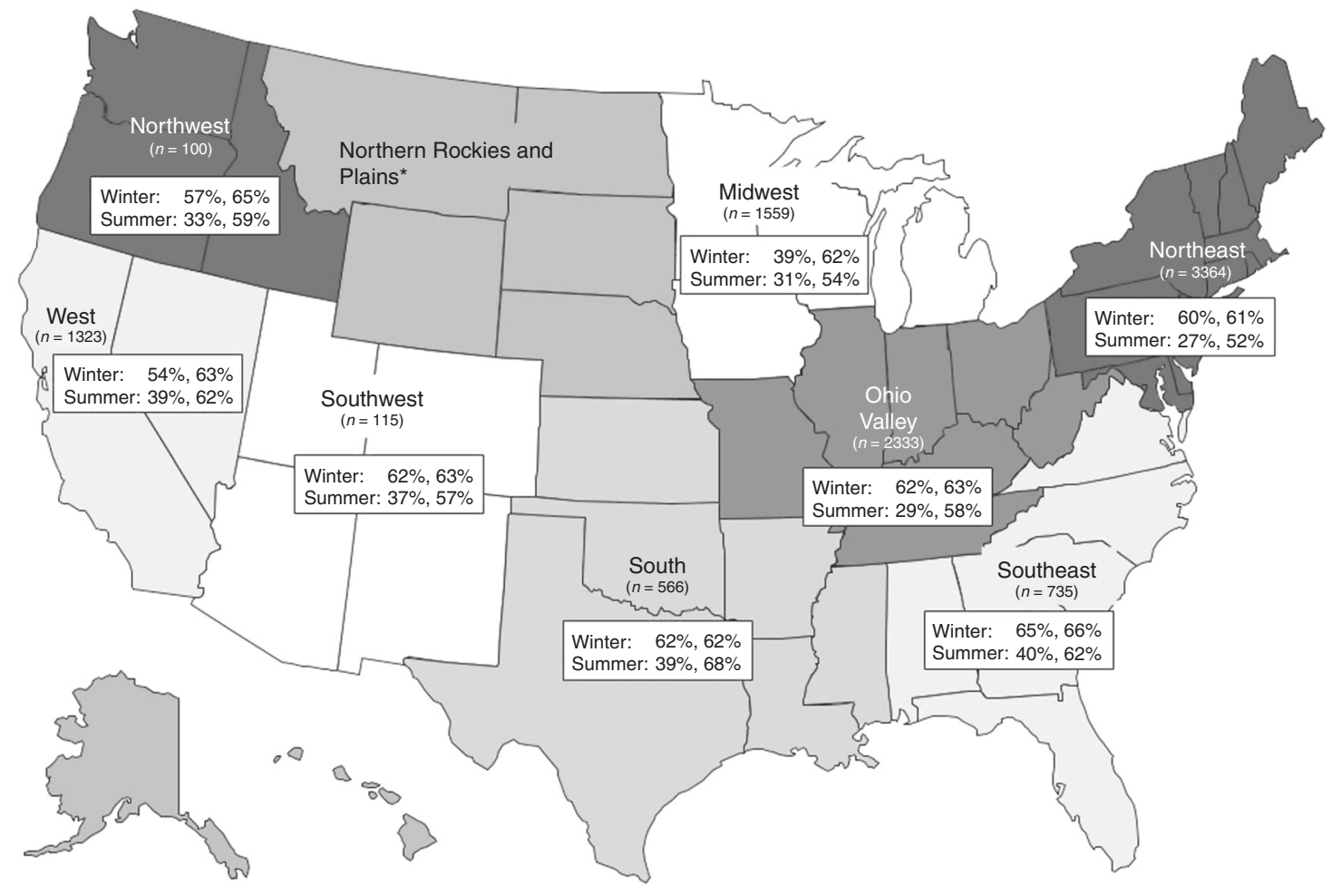

Fig. 2 Adolescents not meeting moderate-to-vigorous and vigorous physical activity recommendations in the winter and summer of 2004 by climate region. Season: $\%, \%$ - Percent of cohort not meeting the MVPA recommendation of $\geq 60$ min per day, percent of cohort not meeting the recommendation of $\geq 3 \mathrm{~h}$ per week VPA, respectively

indicating that season should especially be taken into consideration when measuring PA in younger adolescents.

The impact of gender on seasonal PA levels is not well known. ${ }^{4,26}$ Data from the 2003-2004 and 2005-2006 NHANES have shown that boys are more active than girls in general. ${ }^{28}$ Adjusting for season and age group, the girls in our study were much more likely not to meet MVPA or VPA recommendations than boys were. The gender difference for not meeting the MVPA recommendation was less pronounced in the winter seasons, as compared to the other seasons; this likely reflects that PA levels were lower in the winter across gender, region, and age groups. During the spring, summer, and fall, girls were consistently between 40 and $50 \%$ more likely than boys were not to meet the VPA recommendation.

The lower levels of PA we observed in the fall, winter, and spring seasons provide evidence in support of school-based PA policies and programs to increase adolescent activity levels, especially for adolescent girls. Prior research has demonstrated that physical education programs in school affect adolescent PA levels. Multiple school-based policies, including mandatory physical education, classroom PA breaks, and active commuting to school, all have been found to have larger effects on adolescent MVPA levels than built environment changes. ${ }^{29,30}$ A 2013 Cochran review of randomized controlled trials for school-based PA programs found that school-based interventions had positive effects on increasing the proportion of children and adolescents who engaged in MVPA, as well as on increasing duration of time spent in MVPA. ${ }^{31}$ Our finding of consistently lower levels of meeting MVPA and VPA recommendations in the winter seasons as compared to summer across age group, gender, geographic region, and weight status suggests that adolescents may especially benefit from PA policies and interventions implemented during the school year. Given girls' lower levels of activity as compared to boys during the school year, more research is needed to determine if school-based interventions are more effective when tailored by gender.

Our study has several strengths and limitations. Strengths include the longitudinal, repeated-measures study design spanning different adolescent age groups and developmental stages. We have detailed information collected on activities each season providing a better picture of activities by gender, age group, and season of the year. The large number of participants in the study covers most of the US climate regions and has a similar geographic population density distribution to the US population. However, while geographically diverse, our sample was largely white and is not nationally representative. A second limitation was self-report, which has been found to overestimate PA among adolescents as compared to accelerometer, apart from activities conducted in short bursts, as well as swimming and biking, which may be underestimated. ${ }^{6-8,32}$ To minimize potential overestimation, we excluded walking from our MVPA estimates, swimming, and biking from our VPA estimates.

In conclusion, the majority of adolescents in our study did not meet MVPA or VPA recommendations consistently each season. Adolescents were more at risk for not meeting PA recommendations in the winter than summer regardless of US climate region, gender, ethnicity, or age group. Our findings suggest that schoolbased policies and interventions to promote MVPA and VPA may be particularly beneficial during adolescence.

\section{ACKNOWLEDGEMENTS}

This study was funded by the Breast Cancer Research Foundation and grants (R01DK084001 and DK046200) from the National Institutes of Health. M.L.K. was supported by a training grant T32HD060454 in Reproductive, Perinatal, and Pediatric Epidemiology from the National Institute of Child Health and Human Development, National Institutes of Health. 


\section{ADDITIONAL INFORMATION}

Competing interests: The authors declare no competing interests.

\section{REFERENCES}

1. Barlow, S. E. Expert committee recommendations regarding the prevention, assessment, and treatment of child and adolescent overweight and obesity: Summary report. Pediatrics 120, S164-S192 (2007).

2. Carson, V. \& Spence, J. C. Seasonal variation in physical activity among children and adolescents: a review. Pediatr. Exerc. Sci. 22, 81-92 (2010).

3. Shephard, R. J. \& Aoyagi, Y. Seasonal variations in physical activity and implications for human health. Eur. J. Appl. Physiol. Occup. Physiol. 107, 251-271 (2009).

4. Rich, C., Griffiths, L. J. \& Dezateux, C. Seasonal variation in accelerometerdetermined sedentary behaviour and physical activity in children: a review. Int. J. Behav. Nutr. Phys. Act. 9, 49 (2012).

5. Berkey, C. S. et al. One-year changes in activity and in inactivity among 10- to 15year-old boys and girls: relationship to change in body mass index. Pediatrics 111, 836-843 (2003).

6. Garriguet, D. \& Colley, R. C. A comparison of self-reported leisure-time physical activity and measured moderate-to-vigorous physical activity in adolescents and adults. Health Rep. 25, 3-11 (2014).

7. Adamo, K. B. et al. A comparison of indirect versus direct measures for assessing physical activity in the pediatric population: a systematic review. Int. J. Pediatr. Obes. 4, 2-27 (2009).

8. Harrison, F. et al. Seasonality in swimming and cycling: exploring a limitation of accelerometer based studies. Prev. Med. Rep. 7, 16-19 (2017).

9. Lamonte, M. J. \& Ainsworth, B. E. Quantifying energy expenditure and physical activity in the context of dose response. Med. Sci. Sports Exerc. 33, S370-S378 (2001).

10. Shephard, R. J. Limits to the measurement of habitual physical activity by questionnaires. Br. J. Sports Med. 37, 197-206 (2003).

11. Rich-Edwards, J. W. et al. Adolescent body mass index and infertility caused by ovulatory disorder. Am. J. Obstet. Gynecol. 171, 171-177 (1994).

12. Berkey, C. S. et al. Activity, dietary intake, and weight changes in a longitudinal study of preadolescent and adolescent boys and girls. Pediatrics 105, E56 (2000).

13. Field, A. E. et al. Association of sports drinks with weight gain among adolescents and young adults. Obesity 22, 2238-2243 (2014).

14. Gortmaker, S. L. et al. Television viewing as a cause of increasing obesity among children in the United States, 1986-1990. Arch. Pediatr. Adolesc. Med. 150, 356-362 (1996).
15. Taveras, E. M. et al. Longitudinal relationship between television viewing and leisure-time physical activity during adolescence. Pediatrics 119, E314-E319 (2007).

16. Rifas-Shiman, S. L. et al. Comparing physical activity questionnaires for youth: seasonal vs annual format. Am. J. Prev. Med. 20, 282-285 (2001).

17. Ainsworth, B. E. et al. 2011 Compendium of physical activities: a second update of codes and MET values. Med. Sci. Sports Exerc. 43, 1575-1581 (2011).

18. Greydanus, D. E. \& Bashe, P. American Academy of Pediatrics. Caring for your Teenager: The Complete and Authoritative Guide (Bantam Books, New York, 2003).

19. Strauss, R. S. Comparison of measured and self-reported weight and height in a cross-sectional sample of young adolescents. Int. J. Obes. 23, 904-908 (1999).

20. Cole, T. J. et al. Establishing a standard definition for child overweight and obesity worldwide: International survey. BMJ 320, 1240-1243 (2000).

21. Beighle, A. et al. Seasonality in children's pedometer-measured physical activity levels. Res. Q. Exerc. Sport 79, 256-260 (2008).

22. Belanger, M. et al. Influence of weather conditions and season on physical activity in adolescents. Ann. Epidemiol. 19, 180-186 (2009).

23. Benefice, E. \& Cames, C. Physical activity patterns of rural Senegalese adolescent girls during the dry and rainy seasons measured by movement registration and direct observation methods. Eur. J. Clin. Nutr. 53, 636-643 (1999).

24. Finn, K., Johannsen, N. \& Specker, B. Factors associated with physical activity in preschool children. J. Pediatr. 140, 81-85 (2002).

25. Mattocks, C. et al. Intraindividual variation of objectively measured physical activity in children. Med. Sci. Sports Exerc. 39, 622-629 (2007).

26. Gracia-Marco, L. et al. Seasonal variation in physical activity and sedentary time in different European regions. The HELENA study. J. Sports Sci. 31, 1831-1840 (2013).

27. Armstrong, N. et al. Patterns of physical-activity among 11-year-old to 16-year-old British children. BMJ 301, 203-205 (1990).

28. Chung, A. E. et al. Physical activity and BMI in a nationally representative sample of children and adolescents. Clin. Pediatr. (Phila.). 51, 122-129 (2012).

29. Palakshappa, D. et al. Adolescent physical education class participation as a predictor for adult physical activity. Child. Obes. 11, 616-623 (2015).

30. Bassett, D. R. et al. Estimated energy expenditures for school-based policies and active living. Am. J. Prev. Med. 44, 108-113 (2013).

31. Dobbins M., et al. School-based physical activity programs for promoting physical activity and fitness in children and adolescents aged 6 to 18. Cochrane Database Syst. Rev. 2, CD007651 (2013).

32. Troiano, R. P. et al. Physical activity in the United States measured by accelerometer. Med. Sci. Sports Exerc. 40, 181-188 (2008). 